\title{
Obtención de modelos urbanos tridimensionales
}

\section{Generation of three-dimensional urban models}

\author{
R. Rodríguez ${ }^{(*)}, \underline{\text { M. Álvarez }}{ }^{(*)}$, M. Miranda ${ }^{(*)}$, A. Díez ${ }^{(*)}$, F. Papí(*), P. Rodríguez ${ }^{(* * *}$
}

RESUMEN

El uso de modelos urbanos 3D se extiende cada vez a una mayor diversidad de áreas y aplicaciones, pero su utilización como una herramienta habitual dentro del proceso de realización del proyecto arquitectónico y urbanístico se ve limitada por la lentitud de los procesos de generación de los modelos y el alto coste de realización y mantenimiento de los mismos. Su uso, si el alcance del proyecto lo permite, suele estar encuadrado en propósitos de visualización en la etapa final.

Los autores de este trabajo han desarrollado una metodología para la automatización del proceso de generación de estos modelos de ciudad, que reduce tiempos y costes, y que permitirá una mayor generalización de su uso.

$113-97$

Palabras clave: Modelo urbano 3D; cityGML; LIDAR; automatización; IDE.

\section{SUMMARY}

The use of 3D urban models extends increasingly to a broader range of areas and applications, but its use as a standard tool in the process of realization of the architectural and urban design is limited by the slow pace of the generation processes of models and the derived high cost of realization and maintenance of the models. That is why we find that its use, the size of the project permitting, often framed in purposes of visualization in the final stage.

The authors of this paper have developed a methodology for the automation of the process of generation of these city models, cut time and costs, and that will allow a greater generalization of its use.
Keywords: 3D Urban model; cityGML; LIDAR; automation; SDI.

\footnotetext{
(*) Universidad Politécnica de Madrid (España).

(**) Instituto Geográfico Nacional (España).

(***) Universidad de Málaga (España).

Persona de contacto/Corresponding author: marina.alvarez@upm.es (M. Álvarez)
}

Recibido/Received: 04 may 2012 Aceptado/Accepted: 08 jul 2012 


\section{INTRODUCCIÓN}

Un modelo urbano 3D es una representación digital de la superficie de la tierra y de los objetos de las áreas urbanas. Se puede usar para realizar aplicaciones que permitan, entre otras, la gestión del planeamiento urbano, estudios de contaminación acústica y ambiental, gestión de situaciones de emergencia, etc. Estas aplicaciones deberían ser accesibles para el usuario utilizando formas de visualización de la información comprensibles para la mayor parte de la población.

Otro de los posibles usos de los modelos urbanos podría ser su incorporación en el desarrollo de proyectos arquitectónicos, pero su alto coste económico y su enorme coste temporal los hacen inviables en la mayoría de ellos. La introducción de modelos tridimensionales de la ciudad en las primeras etapas de un proyecto arquitectónico permitiría (entre otras consideraciones) realizar un análisis previo al proyecto urbanístico elaborado con técnicas de toma de decisiones, evaluar la ubicación más apropiada de un edificio, mostrar los cambios temporales en una localización y realizar el análisis del territorio (1). También permitiría analizar el volumen y altura de una nueva edificación con respecto a su entorno, los materiales, estudios de iluminación, adaptación al perfil del terreno y al perfil urbano y en definitiva, el impacto real de la nueva construcción.

La realización de herramientas para la generación de modelos urbanos tridimensionales comenzó hace casi dos décadas, alcanzando su máximo desarrollo a partir de la incorporación de internet a los Sistemas de Información Geográfica (SIG). Por un lado la expansión de la telefonía móvil y por otro lado el deseo de poner a disposición del usuario final tecnologías GNSS (Global Navigation Satellite System) ha producido un auge en la investigación de métodos que conlleven una generación de modelos tridimensionales urbanos de mayor calidad y con un menor coste. El uso actual de aplicaciones y servicios web avanzados requiere modelos urbanos de alta calidad y que puedan además ser desarrollados automáticamente de manera que permitan una rápida obtención de resultados a un precio reducido. Como ejemplo se puede mencionar el desarrollo del modelo 3D de Berlín (·3D BERLIN PROYECT) en el Instituto de Fotogrametría de la Universidad de Stuttgart para la empresa Google (2).

A principios del siglo XXI, empezaron a desarrollarse métodos de extracción de edificios en zonas de alta densidad, aunque con resultados no fieles a la realidad. En el año 2001 se realizó en Phoenix (USA)(3) un intento de implementar una base de datos con cien modelos de ciudades, obtenidos por vectorización de las líneas exteriores de cada edificación. La técnica usada era totalmente manual y la fuente de datos era únicamente la fotogrametría digital. Presentaba el inconveniente de que todas las construcciones tenían una sola altura, debido a la imposibilidad de obtener cotas reales de las edificaciones.

Los primeros intentos de automatización completa que fueron ideados para dar respuesta a la necesidad de una gestión de datos eficiente, se basaban en imágenes aéreas o de satélite y se utilizaban únicamente para diferenciar lo construido y lo no construido. Los resultados obtenidos no eran del todo fiables y requerían de una edición manual posterior que elevaba el coste temporal de tal manera que el tiempo final invertido era superior al obtenido con la realización manual completa del modelo.

A partir de este momento las líneas de investigación sobre la extracción automática de edificaciones se bifurcaron en dos vías; una primera vía (que usaba el método de segmentación de la planta) tenía la ventaja de ser muy eficaz para generar la geometría de las plantas de la edificaciones, pero el gran inconveniente de que las cubiertas generadas no reflejaban la realidad de la construcción (4). La segunda vía combinaba una búsqueda heurística en árbol y una segmentación del Modelo Digital de Superficie (MDS) (3). Este método tenía la ventaja de que la estructura de la cubierta era más fiel a la realidad en detrimento de la exactitud del perímetro de los edificios, y el inconveniente de la inexactitud de los encuentros de las distintas pendientes de las cubiertas.

En los años sucesivos de la primera década del siglo XXI el esfuerzo se concentró en el uso e integración de datos procedentes de distintas fuentes, añadiendo a la fotogrametría otras técnicas como la teledetección, los GNSS, el LIDAR (Light Detection and Ranging), el láser-escáner, etc... Esto lleva consigo la utilización de una ingente cantidad de datos que hace inviable la realización de modelos urbanos por métodos manuales o semiautomáticos, con lo que empiezan a tomar importancia los métodos automáticos que proporcionan un modelo más fiel de la realidad.

A la hora de desarrollar una metodología para la automatización del proceso de generación de un modelo urbano tridimensional, se plantean cuatro problemas principales: 
- La necesidad de integrar información procedente de diferentes fuentes de captura de datos (LIDAR, láser-escáner, fotografías aéreas, imágenes de satélite, etc.). Información que presenta diferentes formatos y estructuras de datos (ortofotos en formato ráster, Modelos Digitales del Terreno en formato ráster y vectorial, elementos vectoriales $3 \mathrm{D}$ y atributos no espaciales asociados con los elementos 3D).

- La necesidad de recoger un enorme volumen de datos, que deben contener una información correcta y veraz de los objetos urbanos. Deben estar georreferenciados y caracterizados por determinadas propiedades como altura, tipo, uso, cubierta, etc.

- La necesidad de utilizar diferentes aplicaciones y programas para generar modelos a resoluciones bajas y un modelo estándar que represente tanto la geometría como la información semántica y que sean interoperables.

- La necesidad de reducir el tiempo y el coste que supone la generación de un modelo que tiene una alta componente manual.

En lo que se refiere a la interoperabilidad entre las aplicaciones, programas y formatos es un problema cuya solución se puede beneficiar del creciente número de soluciones Open Source y de estándares para el intercambio y procesamiento de datos auspiciados por diferentes organizaciones dentro de la filosofía de las Infraestructuras de Datos Espaciales (IDE). Desarrolladas bajo la directiva Infraestructure for Spatial Information in Europe (INSPIRE) (Web 1), las Infraestructuras de Datos Espaciales (IDE) proporcionan un marco adecuado para propiciar el intercambio de datos y satisfacer la interoperabilidad tanto técnica como semántica (5).

En este contexto, el Open Geospatial Consortium (OGC) (Web 2) ha desarrollado estándares para intercambiar y visualizar información 2D; entre otros cabe destacar el Web Map Service (WMS) (Web 3), el Web Feature Server (WFS) (Web 4) y el Geographic Mark Up Language (GML) (Web 5) basado en la gramática de XML.

El GML es un lenguaje utilizado para expresar características geográficas que constituye una capa semántica sobre XML y que proporciona un conjunto de clases que describen los elementos geográficos como entidades, sistemas de referencia espaciales, geometrías, topologías, tiempo, unidades de medida y valores generales. El OGC ha desarrollado también el estándar de intercambio de datos
3D (X3D) y el GML3. En esta misma vía, para la modelización y visualización 3D, la International Standarisation Organisation (ISO) (Web 6) ha desarrollado la familia de Normas 19000.

Desde agosto de 2008 existe un estándar internacional OGC, el CityGML (Web 7), para el intercambio de modelos urbanos entre dos sistemas que utilizan vocabulario o conceptos diferentes y para la realización de modelos urbanos virtuales 3D con datos procedentes de diversas fuentes. Los primeros trabajos sobre el CityGML surgieron en 2002 en Alemania como iniciativa de la GDI NRW (Initiative Geodata Infraestructure North-Rhine Westphalia) dentro de de las IDE.

\subsection{CityGML}

Es un estándar para la realización de modelos urbanos 3D que usa un subconjunto del modelo geométrico de GML3 y una implementación de la Norma ISO 19107 Spatial Schema. Asimismo es un estándar complementario de normas, tanto de gráficos por ordenador 3D (6), VRML (Web 8) y COLLADA (Web 9), como de geovisualización KML (Web 10). A diferencia de KML (Google), CityGML utiliza semántica y geometría estructurada.

Define un modelo de información común (ontología) y dispone de una capa semántica de propósito general característica de la Web Semántica o Web 3.0 (7) (8) (Web 11). Esto permite establecer un alto grado de interoperabilidad técnica para comunicar varios sistemas de procesamiento espacial en tiempo real, usando interfaces compartidas y semántica que proporciona la capacidad de entender el contenido de los datos, su calidad y su significado.

Al ser también un estándar para el intercambio de datos elaborado dentro de la filosofía de las IDE, soporta la integración de datos desde diferentes bases de datos y conjuntos de datos almacenados en sistemas de información de múltiples organismos. Para facilitar la colaboración entre los sistemas de información es preciso diseñar un servicio y un gestor de procesos sobre los modelos 3D que ayude a mejorar las comunicaciones entre los diferentes agentes y asegure la interoperabilidad ente las diferentes herramientas software y plataformas asociadas a sistemas de información en entornos urbanos (9).

CityGML, que proporciona un modelo de datos (UML) (10) para la representación de entornos urbanos 3D, presenta las siguientes características (11) (12): 
1. Niveles de detalle LODs de CityGML. Karlsruhe Institute of Technology. Semantic Data Models (Web 12).
Es un modelo multifunción que permite pasar de una gestión de datos a una interpretación de los mismos. Incluye diferentes funcionalidades, almacenamiento de datos, modelado de bases de datos, intercambio de datos y se puede usar como información de base en Sistemas de Información Geográficos (SIG).

Proporciona mecanismos de extensión para enriquecer el modelo para problemas específicos. También proporciona elementos para representar atributos temáticos, geometrías y topologías 3D, semánticas, taxonomías y agregaciones de datos hasta cinco niveles de detalle LOD's (LOD0, LOD1, LOD2, LOD3, LOD4) (Figura 1). En cada uno de ellos la información debe elaborarse con una determinada precisión y características, de tal manera que pueda adaptarse a la escala de representación que demande cada aplicación:

- LOD 0, modelo regional, es el Modelo Digital del Terreno (MDT) con una precisión de $5 \mathrm{~m}$. No se representa ningún tipo de estructura ni cubierta.

- LOD1, modelo de ciudad, es el modelo básico de edificio (su volumen) y otros elementos urbanos sin cubiertas ni elementos laterales o exteriores con un error no superior a 5 metros. Se representan los objetos mayores de $5 \times 5$ metros.

- LOD2, modelo de ciudad, es el LOD1 con algo más de detalle y con una precisión de 2 metros. Se representan las cubiertas con sus elementos reales y "prototipos" de mobiliario urbano, algo de vegetación, ventanas, puertas, calles, tejados texturizados y diferenciados.

- LOD3, modelo de ciudad, es a nivel de detalle, el modelo arquitectónico exterior con precisión de 0,5 metros. Se representa el mobiliario urbano, las calles y la vegetación con su apariencia real.

- LOD4, Modelo interior, es a nivel de detalle, el modelo interior de los objetos con una precisión de $20 \mathrm{~cm}$.

Un objeto puede ser representado simultáneamente en diferentes LOD en un mismo conjunto de datos, proporcionando la posibilidad de análisis y visualización del mismo elemento en diferentes grados de resolución. Igualmente se pueden combinar e integrar dos conjuntos de datos que contengan el mismo objeto en diferentes LOD; usar múltiples resoluciones permite que los datos semánticos disponibles sean compatibles entre sí.

\subsection{Objetivos}

El objetivo principal del trabajo consiste en obtener un modelo tridimensional en formato CityGML para una zona de la ciudad de Alicante. Las coordenadas del área de trabajo en proyección UTM, sobre el elipsoide WGS84 son las siguientes:

x: 719692-720172

$y: 4247015-4247496$

La consecución de este objetivo principal implica el desarrollo de los siguientes objetivos secundarios:

- Generación de un Modelo Digital del Terreno (MDT) y hacer una clasificación de los puntos disponibles.

- Generar los volúmenes exteriores de los edificios y la forma de las cubiertas.

- Generar escenarios 3D usando el formato CityGML.

- Realizar un control de calidad mediante la comparación con modelos estereoscópicos clásicos y restitución fotogramétrica.

- Disminuir los plazos de desarrollo y los costes de realización de un modelo 3D frente a las técnicas tradicionales, con la búsqueda de un alto nivel de automatización.

- Conseguir interoperabilidad con las aplicaciones de realidad virtual existentes en el mercado y obtener gran versatilidad en cuanto a extensión y tipología del entorno urbano.

\section{METODOLOGÍA}

Los autores de este trabajo han desarrollado una metodología de desarrollo de un modelo urbano basado en las siguientes premisas:
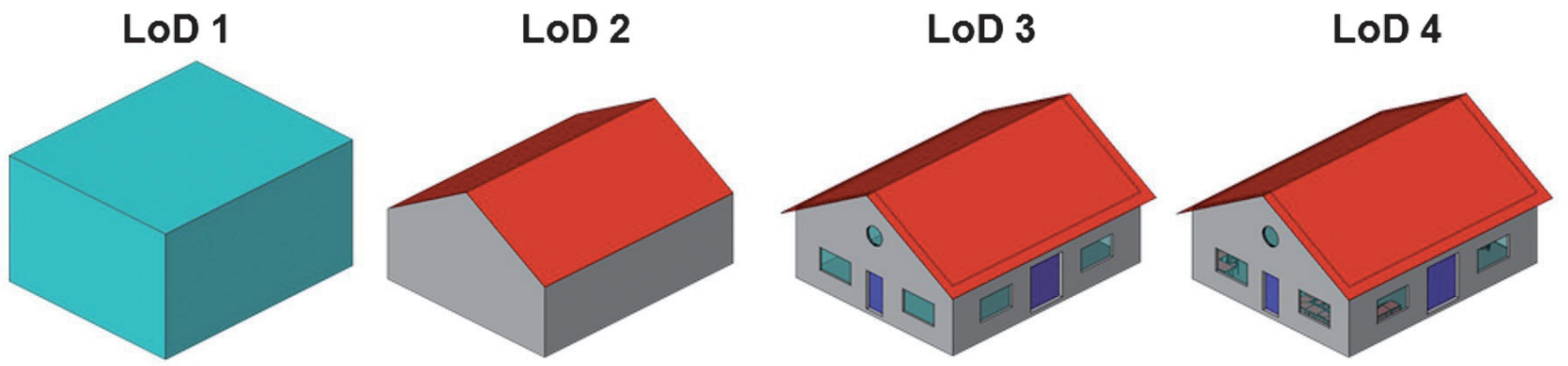
1. Integración de los datos proporcionados por imágenes de alta resolución con datos obtenidos a través de sensores LIDAR (13). Es una tecnología que permite determinar la distancia a un objeto o superficie utilizando un haz láser pulsado emitido desde un sensor activo aerotransportado que no requiere de condiciones de visibilidad especiales para la obtención de los datos (14). El resultado es una nube de puntos que, además de las coordenadas tridimensionales de cada punto, proporciona datos que permiten conocer, entre otros, la naturaleza del material (nivel de intensidad).

2. El desarrollo de un método propio automático de clasificación de los puntos.

3. La utilización del estándar CityGML.

El proceso comprende cuatro etapas consecutivas, que a su vez se descomponen en varias fases:

1. Recopilación de la Información.
2. Creación de escenarios 3D.

2.1. Generación del MDT.

2. Diagrama de flujo del proceso completo.

Modelado de la Información 3D.

2.3. Implementación de la Base de Datos.

3. Control de calidad.

4. Desarrollo de un visualizador Web.

La arquitectura del proceso se muestra en el diagrama de flujo de la Figura 2.

\section{DESARROLLO DE LA APLICACIÓN}

En este trabajo se exponen los resultados obtenidos hasta el momento en el desarroIlo de los niveles LOD0, LOD1 y LOD2 del proceso de modelización espacial.

\subsection{Recopilación de la Información}

Para el desarrollo del trabajo se ha contado con los siguientes datos:

- Datos en formato vectorial, en formato DXF de la D. G. del Catastro (Web 13).

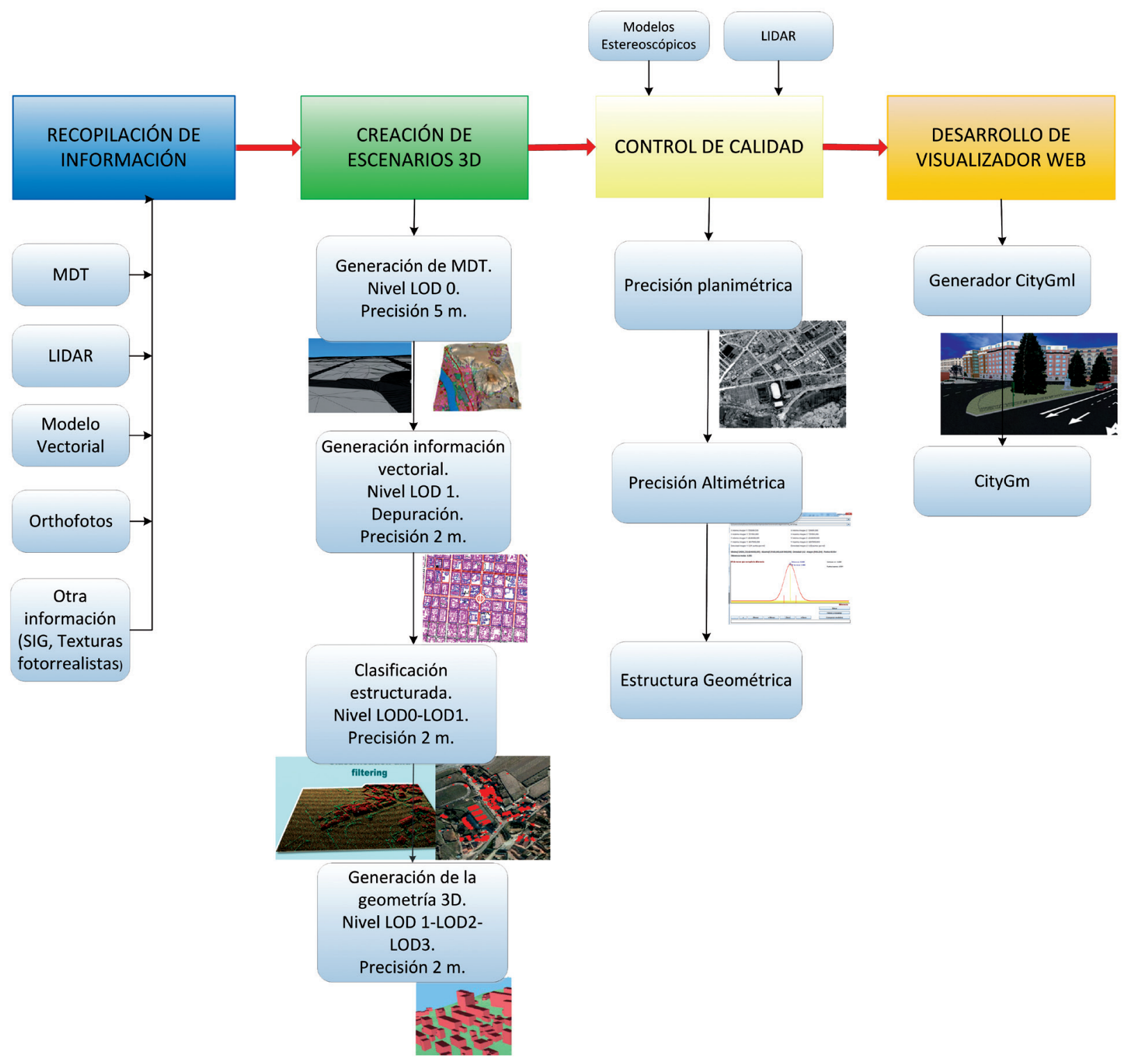


3. Creación de escenarios 3D.
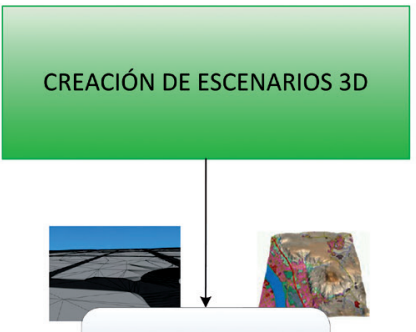

Generación de MDT.

Nivel LOD 0.

Precisión $5 \mathrm{~m}$.

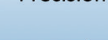

Directo del MDT
- Imágenes, necesarias a la hora de ubicar visualmente los datos y entender el contexto en el que son presentados. Se han utilizado ortofotos del Plan Nacional de Ortofotografía Aérea (PNOA) del IGN (Web 14).

- Modelos digitales del terreno con paso de malla de $5 \mathrm{~m}$, en formato $\mathrm{XYZ}$ proporcionado por el IGN (Web 15).

- Nube de puntos procedentes del sensor LIDAR, con una densidad de 2 puntos $/ \mathrm{m}^{2}$ proporcionado por el IGN.

Toda al información ha sido tratada y editada con el siguiente software: ArcGIS, para el tratamiento de toda la información (Web 16); DIGI3D/MDTop, para el tratamiento de datos fotogramétricos; TerraSCAN, Software para tratamiento de la información LIDAR.

\subsection{Creación de escenarios 3D}

El proceso de creación de los escenarios, que se muestra en la Figura 3, se ha desarrollado en las siguientes fases:

1. Generación del MDT, LOD0.

2. Modelado de la Información 3D.

2.1. Generación del LOD 1.

2.2. Generación del LOD 2.

3. Implementación de la Base de Datos.

\subsubsection{Obtención del MDT, LOD 0}

En CityGML el terreno, LOD 0 se puede especificar como un Modelo Digital del Terreno (MDT) en forma TIN (Triangulated Irregular Network) obtenido a partir de datos LIDAR.

El cálculo de MDT con datos LIDAR no es un proceso inmediato, ya que la obtención de datos registra mucha información geográfica, que debe ser filtrada y clasificada, siendo el principal problema la selección de puntos que definen la superficie del terreno. La obtención del MDT depende de varios factores:

- La densidad de elementos geográficos con altura que exista en la zona de estudio, que impiden, en la mayoría de los casos (edificios, presencia de vegetación cerrada), que los haces de energía emitidos Ileguen al suelo.

- La variación de la pendiente del terreno.

- El tamaño de los objetos.

En este trabajo se usó una metodología propia que integra datos procedentes de sensores LIDAR y datos proporcionados por imágenes de alta resolución, para obtener un MDT con precisión de $5 \mathrm{~m}$, realizando una clasificación automatizada de los puntos.

Se usó el clasificador de máxima verosimilitud ML (Maximun Likelihood) (15), adecuado para resolver el problema cuando se usan muchas bandas de información espectral y otros atributos de forma simultánea (16). Se han desechado otros clasificadores como el de Mínima Distancia y el de los Paralelepípedos, por considerarlos inadecuados para este trabajo. El primero, en el que a cada punto se le asigna la categoría o clase más cercana, se usa generalmente por su sencillez cuando se utilizan pocas bandas de información (en nuestro caso disponemos de bastantes bandas de información); el segundo, que implica prefijar un área de dominio para cada clase, tiene el inconveniente de que aparecen puntos asignados a varias clases o también aparecen puntos sin clasificar (en nuestro caso, requerimos que todos los puntos sean clasificados y en una única clase).

El método ML considera como hipótesis de partida que las clases que se desean obtener se distribuyen con la misma probabilidad en la imagen considerada; esto no siempre es así, por lo que dicho método se mejora y se amplía con el conocido como método de decisión Bayesiano (BY) (17). Este asigna una probabilidad de ocurrencia diferente a cada clase (18) (19) 20). Se ha demostrado que, modificando las probabilidades a priori en el clasificador ML para obtener el clasificador BY, se puede mejorar la precisión del resultado obtenido para aquellas clases con bandas espectrales similares, sin afectar la decisión de clasificación para aquellas clases que presentan bandas espectrales muy definidas (15) (19).

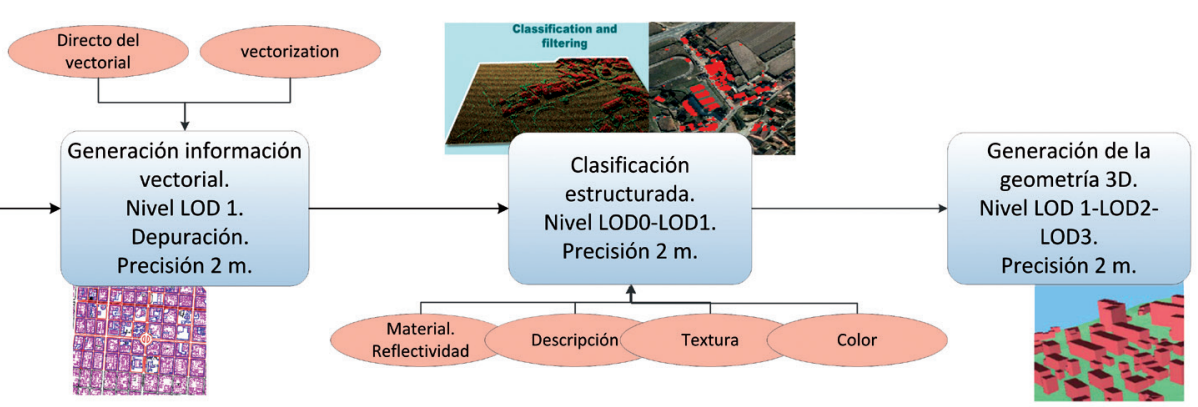


El proceso de construcción del MDT y posterior clasificación de elementos constó de las siguientes fases:

- Se fusionó la información proporcionada por el LIDAR con la información proporcionada por las imágenes de alta resolución (color a 8 bits y pancromático a 16 bits), para hacer corresponder a cada punto LIDAR un valor digital (VD) en formato HSI (Hue, Saturation, Intensity) transformado a partir del RGB (Red, Green, Blue) a fin de separar la información de color obtenida de los atributos $\mathrm{H}$ y $\mathrm{S}$ (tono y saturación) de la información obtenida del atributo I (blanco y negro); este último atributo se sustituyó por la información proporcionada por la cámara pancromática que presentaba una resolución mayor (16 bits para la información de blanco y negro).

- Para mejorar los resultados de clasificación que se obtendrían usando los atributos anteriores, a esta información espectral se le añadió:

- La componente $\mathrm{R}$ de la cámara infrarroja, codificada a 8 bits, que es linealmente independiente de los atributos anteriores $(\mathrm{H}, \mathrm{S}, \mathrm{I})$.

- El nivel de intensidad LIDAR codificado a 8 bits.

- El incremento de Z del punto, es decir la diferencia de $Z$ que hay entre el primer y último pulso LIDAR (p.e. en una zona de cubierta vegetal, el primer pulso LIDAR corresponde a la copa de los árboles y el último pulso LIDAR corresponde al terreno).

Esto hace que la clasificación de los puntos LIDAR, se realice en total con 6 atributos independientes o muy poco correlados $(\mathrm{H}, \mathrm{S}, \mathrm{I}$, infrarrojo, intensidad LIDAR incremento de Z) (14):

- Mediante el algoritmo de Máxima Verosimilitud (ML), se clasificaron los puntos del LIDAR con sus atributos en varias clases principales (puntos en edificios, puntos en el terreno, puntos en cobertura vegetal, puntos en calles, etc.).

- Tomando sólo los puntos sobre el terreno, se realizó un proceso de triangulación de Delaunay (21) para obtener un MDT en forma de TIN (Triangulated Irregular Network) con una precisión inferior a 5 metros. El resultado se muestra en la Figura 4.

\subsubsection{Modelado de información 3D}

El modelo de edificios es el núcleo de CityGML ya que permite la representación temática y espacial de los edificios (sus partes y accesorios) en los cuatro niveles de detalle mencionados anteriormente (LOD's).

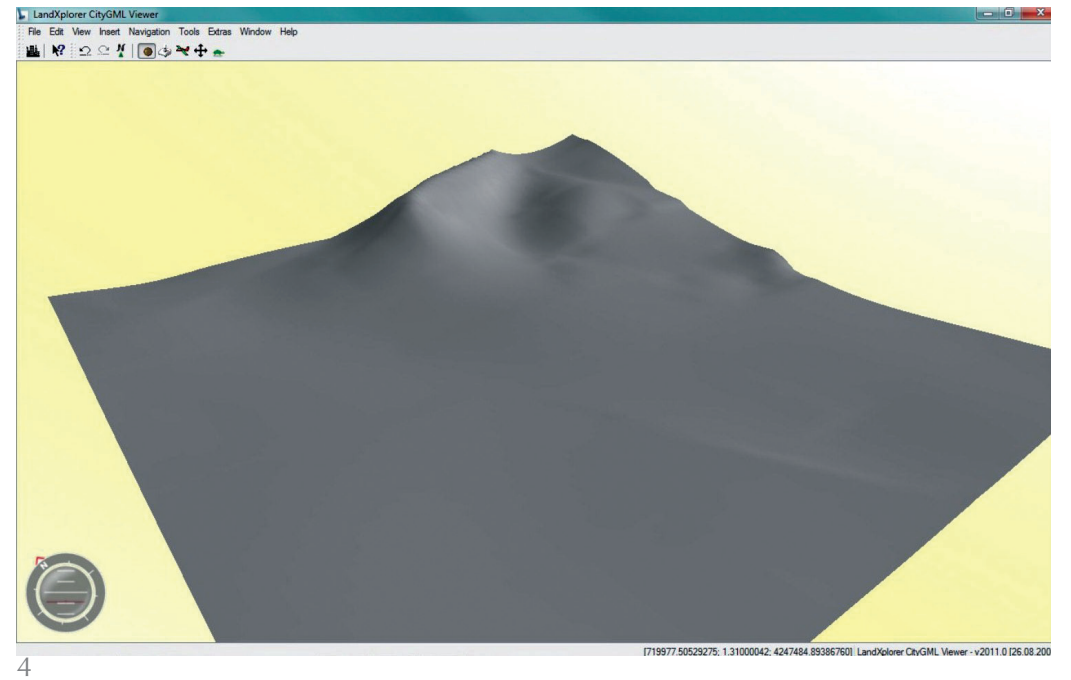

La realización de un modelo se debe ajustar a una serie de premisas:

- Todas las coordenadas deben pertenecer 4. Modelo Digital del Terreno (MDT) obtenido visualizado en LandXplorer a un sistema de coordenadas de carácter global, no permitiéndose transformaciones locales (en nuestro caso, hemos usado la proyección UTM sobre elipsoide WGS84).

- A los elementos geográficos, de acuerdo a la Norma ISO 19109, se le pueden asignar más de una propiedad espacial.

- El modelo topológico debe seguir la Norma ISO 19107 y GML3 (Web 17). Las primitivas nodo, eje, cara, sólido y agregaciones deben satisfacer un número de reglas de integridad que garanticen la consistencia del modelo sin ninguna redundancia.

- La geometría de los elementos geográficos debe seguir la Norma ISO 19107 y GML3.

- Las propiedades espaciales de los objetos temáticos deben ser representadas por el modelo geométrico-topológico Boundary Representation (B-Rep) (6).

- La información sobre la apariencia de las superficies se considera como parte integral de los modelos urbanos 3D virtuales y se añade a las propiedades semánticas y espaciales.

- El interior de los edificios se modela mediante habitaciones. Se utiliza la teoría de grafos para representar las relaciones de adyacencia.

A continuación se presenta la implementación de los niveles LOD1 y LOD2 (17) (22).

\section{1. ${ }^{a}$ Generación del LOD1}

Como se mencionó anteriormente en el nivel LOD1, se construyen los volúmenes de los edificios y otros elementos, a partir de la información existente y de forma automática, con precisión $2 \mathrm{~m}$. En las Figuras 5 a 9 se presenta el resultado del proceso que se desarrolla en las siguientes fases: 
5. Planta de los edificios en 2D visualizada en la aplicación de generación automática desarrollada.

6. Nube de puntos LIDAR con los puntos que caen dentro de los edificios en azul, visualizada en la aplicación de generación automática desarrollada.
- A partir de la clasificación obtenida en el desarrollo del MDT del LOD0, se toman los puntos pertenecientes a los edificios, terreno y calle y se genera un nuevo Modelo Digital de Superficie clasificado y sin otros elementos que las construcciones.

- A partir de él se vuelve a generar un TIN, del que se seleccionan los triángulos que tienen una pendiente mínima determinada que se establece como umbral, para definir las líneas que forman las plantas de los edificios.

- Usando algoritmos de vectorización y combinando con la información catastral (si estuviese disponible), se obtiene un archivo vectorial bidimensional de la planimetría de los edificios de la zona de trabajo (Figura 5).

- Para realizar la selección automática de los puntos pertenecientes (que caen dentro) de las edificaciones que se han descrito previamente, se sigue el siguiente proceso: - La planta de cualquier elemento clasificado como edificio, se divide en polígonos.

- A continuación, se separan los polígonos cóncavos de los convexos (a través del acimut de los lados).

- Los cóncavos se dividen sucesivamente en dos polígonos hasta que todos los polígonos divididos son convexos, de forma que no queden polígonos cóncavos dentro de la planta de cada edificación. Se calcula el centro de masas de cada uno de los polígonos finales.

- Usando el centro de masas, se divide cada uno de los polígonos en triángulos.

- Se establece que un punto pertenece al polígono (y por tanto a la planta del edificio) si dicho punto pertenece a alguno de sus triángulos (ver puntos azules en la Figura 6).

- Después, se comprueba cuál es la cota 0 de los edificios mediante el análisis de los puntos del último MDT obtenido, siguiendo un proceso de clasificación automática (Figura 7) y descrito a continuación:

- Se comprueban todos los puntos que caen dentro de la planta del edificio y que también pertenecen al MDT.

- Se realiza un análisis estadístico de la distribución de las cotas; analizando su media, su desviación estadística, sus mínimos y sus máximos.

- Del análisis de resultados se obtienen las cotas de los puntos en el terreno en el que se apoya el edificio (p.e. si la desviación estadística es muy pequeña y la cota mínima es próxima a la media el terreno es plano).

- Con la superposición de la ortofotografía se comprueba la fidelidad del resultado obtenido (Figura 8).

- Por último y una vez calculada la cota 0 , se obtienen las alturas de las edificaciones realizando un nuevo análisis de densidad de probabilidad, en este caso, de las alturas dadas por los puntos LIDAR situados en el interior de los límites edificados obtenidos y no del MDT como en el paso anterior (Figura 6).

El modelo tridimensional obtenido y codificado en CityGML para el nivel de detalle LOD 1, visualizado en el visor LandXplorer de Autodesk. (Web 18), se muestra en la Figura 9.

\section{2. ${ }^{a}$ Generación del LOD2}

En este nivel los edificios se presentan con sus cubiertas correspondientes. Para generar el LOD2 con una precisión de $2 \mathrm{~m}$, se realiza un proceso que usa la información de origen y la ya obtenida para el LOD1 y cuyo desarrollo consta de las siguientes fases:

- Se descompone en células simples la planta de las edificaciones generadas en
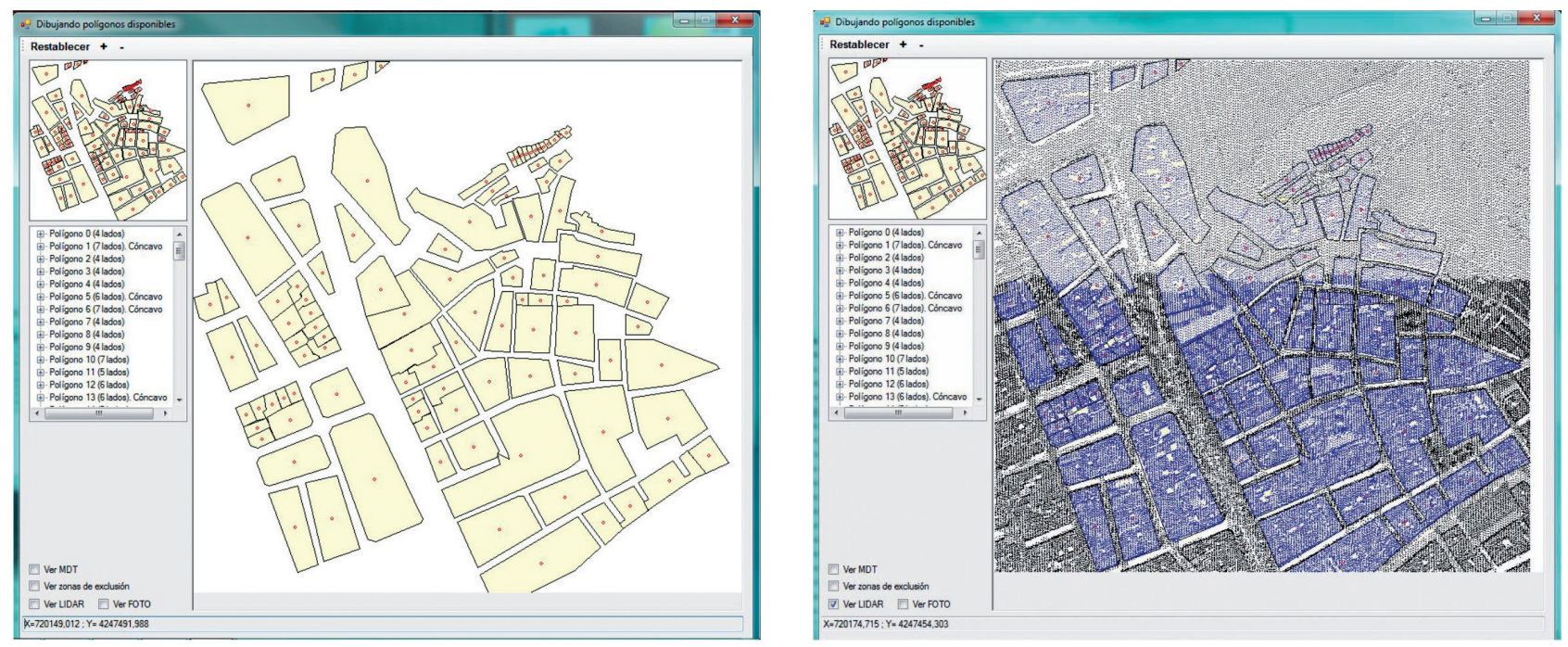

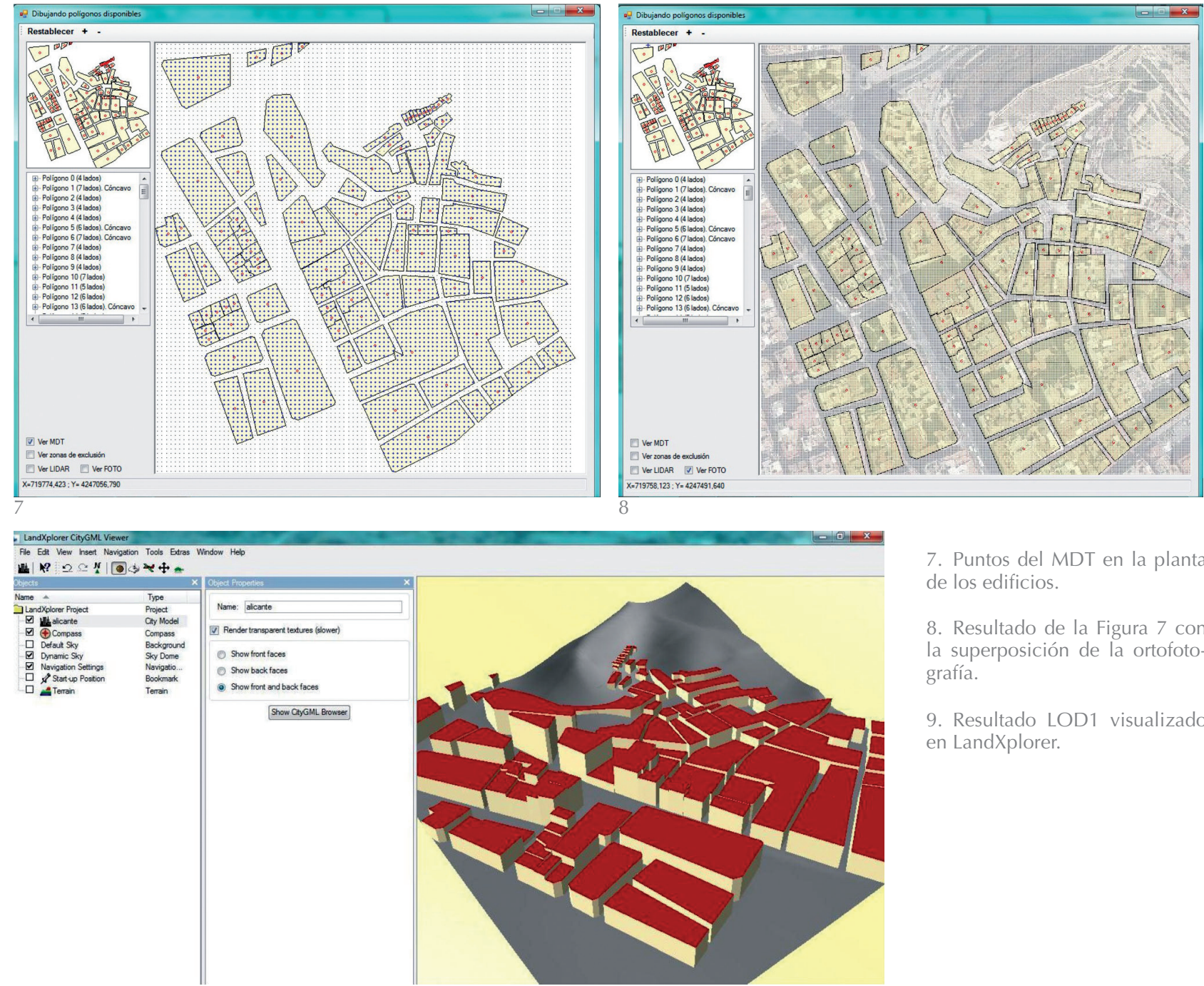

7. Puntos del MDT en la planta de los edificios.

8. Resultado de la Figura 7 con la superposición de la ortofotografía.

9. Resultado LOD1 visualizado en LandXplorer.

2D, de modo que minimicen la variedad de puntos a analizar (17).

- Se analizan las pendientes de la cubierta mediante una triangulación de Delaunay (21) usando los puntos LIDAR que caen dentro de los edificios y que no pertenecen al MDT.

- Se divide la cubierta de cada célula en el número de pendientes distintas proporcionadas por el análisis anterior (17).

- Definidas las cubiertas de cada una de las células, se colocarán en su lugar en planta para la generación de la cubierta completa del edificio (22).

\subsubsection{Implementación de la base de datos 3D}

Aunque en este momento toda la información esta almacenada en un archivo CityGML, se está implementando una base de datos que integrará la información generada en los distintos niveles empleados. Para almacenar CityGML normalmente se utilizan dos bases de datos: en una se almacena la ontología, y en otra se almacena la información semántica y espacial. Esta puede ser un SGBD comercial como Oracle 11G Spatial (Web 19), u OGC como PostgreSQL con la extensión PostGIS activada (Web 20). Esta última es la que se utiliza en este trabajo.

\subsection{Control de calidad}

Se ha realizado un control de calidad en laboratorio mediante la comparación con modelos estereoscópicos clásicos y restitución fotogramétrica, cuyo esquema se puede observar en la Figura 10. De este análisis se comprueba que los errores producidos en el MDT, en la planta de los edificios y en las alturas de los mismos entran dentro de la tolerancia permitida para los diferentes niveles LOD's alcanzados en el proceso de generación automática descrito.

\subsection{Desarrollo de un visualizador web}

Toda la información generada en la construcción del modelo 3D con CityGML debe ser distribuida, bien vía intranet o en internet utilizando un visor web (Figura 11) basado en una arquitectura cliente-servidor. Su realización implica la implemen- 
10. Metodología para el control de calidad de los resultados.

11. Desarrollo visualizador web. 12. Visor web Aristóteles.
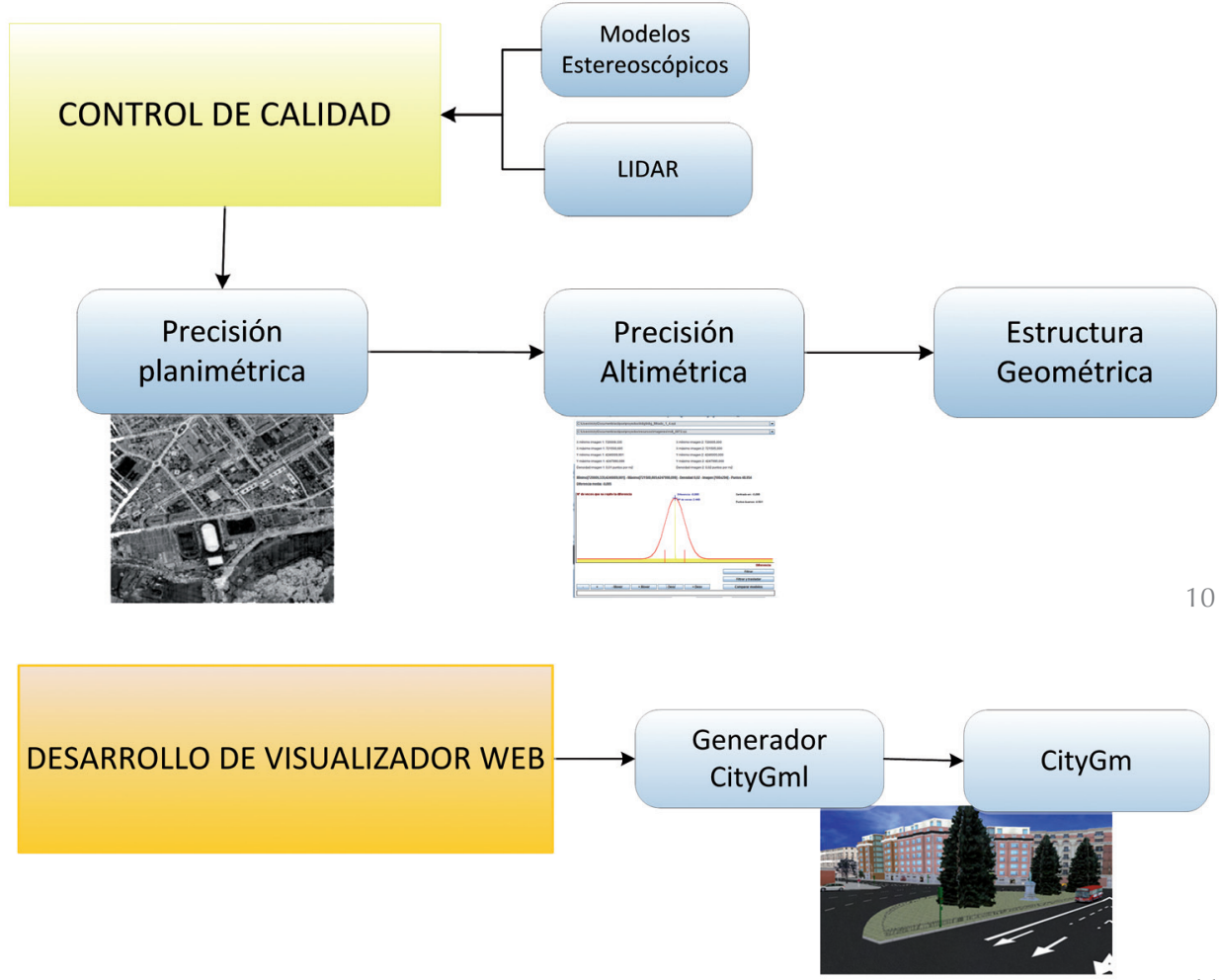

11

tación de un servidor Web, y de un servidor geográfico usando las herramientas de desarrollo Eclipse (Web 21) y C\#.

En la actualidad se está implementando un visor, basado preferentemente en software libre, que permita compatibilidad con otros visores más utilizados como Mapserver (Web 22) y Geoserver (Web 23).

A este fin, se han estudiado distintos visores que permiten visualizar datos en $3 \mathrm{D}$ y que soporten CityGML; comerciales como LandXplorer (C++) de Autodesk (Web 15) o de software libre, como FZKViewer, GML Viewer, BIMserver. En la Figura 12 se mues- tra una imagen del visor elegido, Aristóteles 3D-Wiewer (Java) (Web 24).

\section{CONCLUSIONES}

Los autores han diseñado y desarrollado una metodología propia de generación automática de modelos urbanos de un polígono de la ciudad de Alicante, utilizando las prestaciones del estándar CityGML, a partir de los datos obtenidos de imágenes fotogramétricas, planos 2D vectoriales y datos proporcionados por sensores LIDAR.

En este trabajo se exponen los resultados obtenidos en el desarrollo de los niveles

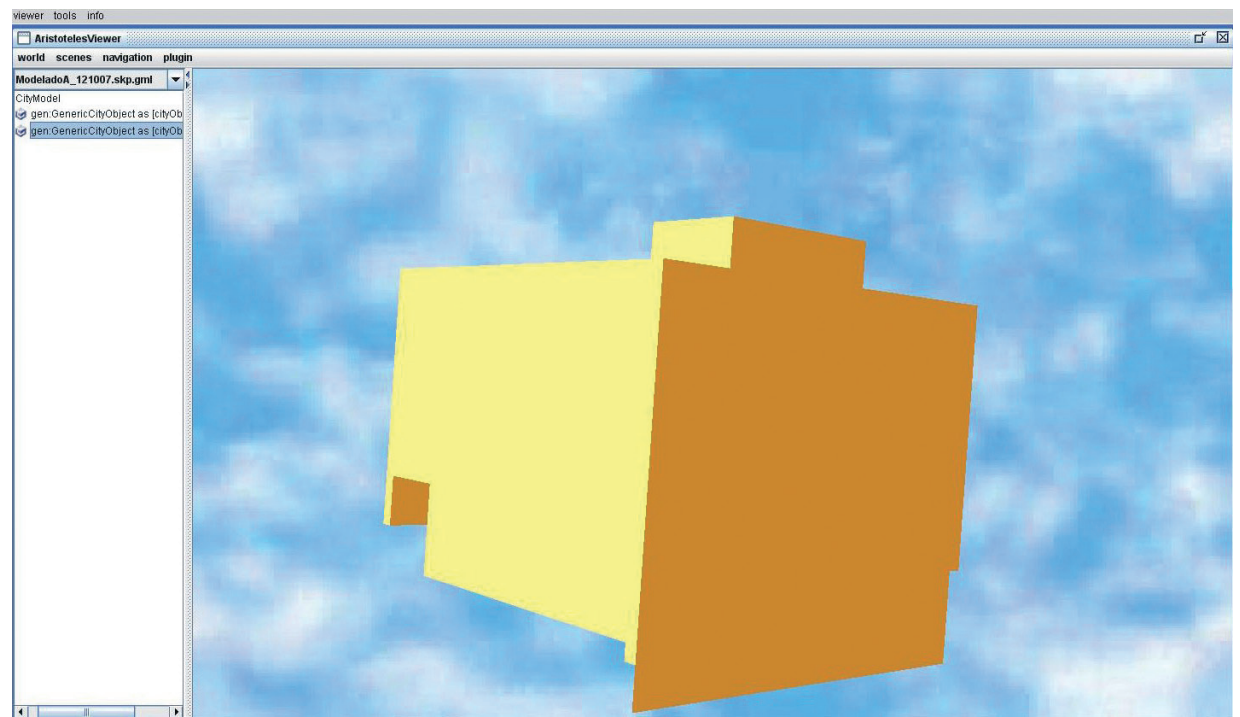


LOD0 y LOD1 del proceso de modelización espacial. Toda la información se ha almacenado en un archivo en formato CityGML. En la actualidad esta en fase de realización el nivel LOD2 y la implementación de una base de datos espacial 3D que integre la información generada de los distintos niveles de detalle característicos del CityGML empleados.

En cuanto al algoritmo de clasificación utilizado, se ha realizado una mejora al clasificador de Máxima Verosimilitud con el método de decisión Bayesiano. Asimismo se ha realizado la clasificación de puntos con 6 atributos independientes entre los que se ha añadido ( $y$ esto supone una novedad con respecto a otros métodos de clasificación) el incremento de $\mathrm{Z}$ dado por los datos del LIDAR (característica geométrica y no radiométrica).

Este trabajo, enmarcado dentro del ámbito de colaboración de la Universidad Politécnica de Madrid con el Instituto Geográfico Nacional, supone un avance en la disminución de los plazos de desarrollo y los costes de realización de un modelo 3D frente a las técnicas tradicionales, y se consigue interoperabilidad con las aplicaciones de realidad virtual del mercado, obteniendo una gran versatilidad en cuanto a extensión y tipología del entorno urbano.

También se pretende conseguir la necesaria fidelidad con el entorno real que repre- senta, en apariencia visual y localización espacial.

La implementación de la solución ha sido realizada siguiendo un enfoque modular que, junto a la arquitectura del sistema, garantizan que la solución sea escalable y accesible desde diferentes tipos de dispositivos con diferentes prestaciones.

Se han realizado ensayos de visualización de los escenarios utilizando el software libre Aristóteles y el software comercial LandXplorer de AutoDesk, que han parecido los más adecuados para la publicación web de resultados en consonancia con la búsqueda de la interoperabilidad, dentro de la filosofía de las IDES.

Se ha comprobado que con la automatización del proceso de generación se aumenta la velocidad de realización del modelo y provoca un aumento de los tiempos dedicados al control de calidad.

Se pretende, asimismo, contribuir al desarrollo y la mejora del estándar CityGML admitido por el Eurosdr a través de los grupos de investigación abiertos.

\section{AGRADECIMIENTOS}

Al Instituto Geográfico Nacional (IGN), por su financiación al proyecto y por suministrar la mayor parte de los datos (fotografías, LIDAR, MDT, etc.).

\section{REFERENCIAS}

(1) Martínez, E., Álvarez, M., Arquero, A., Romero, M. (2010). Apoyo a la selección de emplazamientos óptimos de edificios. Localización de un edificio universitario mediante el Proceso Analítico Jerárquico (AHP) Informes de la Construcción, 519(62): 35-45. doi:10.3989/ic.08.052.

(2) Kada, M. (2009). The 3D Berlin Proyect. Fritsch, D. (ed.): Photogrammetric Week '09, Wichmann Verlag, Heidelberg.

(3) Brenner, C. (2001). City Models-Automation in research and practice. Fritsch/Spiller (eds.): Photogrammetric Week '01, Herbert Wichmann Verlag, Heidelberg.

(4) Brenner, C. (2000). Towards Fully Automatic Generation of City Models. IAPRS Vol. XXXIII, Part B3/1, Comm. III, ISPRS Congress, Amsterdam.

(5) Groot, R., McLaughlin, JD. (2000). Geospatial Data Infrastructure-Concepts, Cases, and Good Practice. Oxford University Press.

(6) Foley, J., van Dam, A., Feiner, S., Hughes, J. (1990). Computer Graphics: Principles and Practice, Second Edition, Addison-Wesley, Reading, Massachusetts.

(7) Finat, J., et al. (2010). Una aproximación semántica a sistemas de información 3D para la resolución de problemas de accesibilidad en patrimonio construido, ACE: Architecture, City and Environment, 13.

(8) Antoniou, G., Van Harmelen, F. (2004). A Semantic Web Primer. Massachusetts Institute of Technology, Londres.

(9) Hundler, J. (2001). Agents and semantic Web. IEEE Intelligent Systems, 16(2), Mar. /Apr.

(10) Booch, G., Rumbaugh, J., Jacobson, I. (1997). Unified Modeling Language User Guide. Addison-Wesley.

(11) Kolbe, TH., Grogër, G. (2003). Towards unified 3D city models, Schiewe, J., Hahn, M, Madden, M, Sester, M (eds): Challenges in Geospatial Analysis, Integration and Visualization, II. Proc. of Joint ISPRS Workshop, Stuttgart. 
(12) Kolbe, TH., Gröger, G. (2004). Unified Representation of 3D City Models. Geoinformation Science Journal, 4 (1).

(13) Ambercore LIDAR (2008). A White Paper of Lidar Mapping [En línea] Disponible en: http://www.ambercore.com/files/TerrapointWhitePaper.pdf.

(14) Díez, A., Arozarena, A., Ormeño, S., Aguirre, J., Rodríguez, R., Saenz, A. (2008). Integración y optimización de tecnologías y metodologías Lidar y fotogramétricas para la producción cartográfica. Proceedings of The international archives of the photogrammetry, remote sensing and spatial information sciences, ISPRS congress Beijing.

(15) Mather, P.M. (1985). A computationally-efficient maximum-likelihood classifier employing prior probabilities for remotely-sensed data. International Journal of Remote Sensing, 6.

(16) Tso, B., Mather, P.M. (2001). Classification Methods for Remotely Sensed Data, Taylor \&.Francis, Londres.

(17) Rodríguez, R., Álvarez, M., Miranda, M., Díaz, A., Papí, F. (2011). Automatic Generation of 3D Virtual Cities from Lidar Data and High Resolution Images. Proceedings of Ist Panel Symposium Emerged/Emerging "disruptive" Technologies, Madrid.

(18) Swain, P.H., Davis, S.M. (1978). Remote Sensing: The Quantitative Approach, McGrawHill, New York.

(19) Strahler, A.H. (1980). The use of prior probabilities in maximum likelihood classification of remotely sensed data. Remote Sensing of Environment, 10: 135-163.

(20) Hutchinson, C.F. (1982). Techniques for combining Landsat and ancillary data for digital classification improvement. Photogrammetric Engineering and Remote Sensing, 48.

(21) Lee, D. T., Schachter, B. J. (1980). Two Algorithms for Constructing a Delaunay Triangulation. Int. J. Computer Information Sci, 9.

(22) Kada, M. (2007). 3D Building Generalisation by Roof Simplification and Typification. Proceedings of the 23th International Cartographic Conference, Moscu.

\section{WEBS:}

(Web 1) Página oficial del proyecto INSPIRE: http://www.inspire-geoportal.eu/

(Web 2) Página oficial de Open Geospatial Consortium: http://www.opengeospatial.org/

(Web 3) Especificaciones estándares para WMS: http://www.idee.es/resources/Servicios/ RinconDesarrollador/RD_wms_v1_3.pdf

(Web 4) Especificaciones estándares para WFS: http://www.idee.es/resources/Servicios/ RinconDesarrollador/RD_wfs_v1_1_0.pdf

(Web 5) Página de GML: http://www.opengeospatial.org/standards/gml

(Web 6) Página de ISO: http://www.iso.org

(Web 7) Página de CityGML OGC Standard Specification: http://www.opengeospatial. org/standards/citygml

(Web 8) Página de WRML: http://www.wrml.org

(Web 9) Página de COLLADA: http://www.collada.org

(Web 10) Página de KML: code.google.com/intl/es/apis/kml/

(Web 11) Página de la Web.3.0: http://web30websemantica.comuf.com/

(Web 12) Página del Kalsruhe Institute of Tecnology: http://www.iai.fzk.de/www-extern/ index.php? $\mathrm{id}=4 \& \mathrm{~L}=1$

(Web 13) Página oficial de la D. G. Catastro-Gobierno de España: https://www.sedecatastro.gob.es/

(Web 14) Página oficial del Plan Nacional de Ortofotografía Aérea (PNOA) del IGN -Gobierno de España: http://www.ign.es/ign/layoutln/actividadesFotoTelePNOA.do

(Web 15) Página oficial del IGN - Gobierno de España: http://www.ign.es/ign/main/index.do

(Web 16) Página oficial de ArcGIS: http://www.esri.com/

(Web 17) Página de GML OGC Standard Specification: http://www.opengeospatial.org/ standards/gml

(Web 18) El producto Autodesk: http://www.autodesk.es/adsk/servlet/ home?sitelD $=455755 \&$ id $=458320$

(Web 19) Página oficial de ORACLE: http://www.oracle.com/index.html

(Web 20) Página de PostGree; http://www.postgresql.org/

(Web 21) Página de Eclipse: http://www.eclipse.org/

(Web 22) Página oficial de la documentación de MapServer: http://mapserver.org/documentation.html

(Web 23) Página oficial de la documentación de GeoServer: http://docs.geoserver.org/

(Web 24) Página de Aristóteles: http://www.ikg.uni-bonn.de/forschung/aristoteles.html 